\title{
Near packings of graphs
}

\author{
Andrzej $\dot{Z} \mathrm{ak}^{*}$ \\ Faculty of Applied Mathematics \\ AGH University of Science and Technology \\ Kraków, Poland \\ zakandrz@agh.edu.pl
}

Submitted: Dec 18, 2012; Accepted: May 16, 2013; Published: May 24, 2013

Mathematics Subject Classifications: 05C70

\begin{abstract}
A packing of a graph $G$ is a set $\left\{G_{1}, G_{2}\right\}$ such that $G_{1} \cong G, G_{2} \cong G$, and $G_{1}$ and $G_{2}$ are edge disjoint subgraphs of $K_{n}$. Let $\mathcal{F}$ be a family of graphs. A near packing admitting $\mathcal{F}$ of a graph $G$ is a generalization of a packing. In a near packing admitting $\mathcal{F}$, the two copies of $G$ may overlap so the subgraph defined by the edges common to both copies is a member of $\mathcal{F}$. In the paper we study three families of graphs (1) $\mathcal{E}_{k}$ - the family of all graphs with at most $k$ edges, (2) $\mathcal{D}_{k}$ - the family of all graphs with maximum degree at most $k$, and (3) $\mathcal{C}_{k}$ - the family of all graphs that do not contain a subgraph of connectivity greater than or equal to $k+1$. By $m(n, \mathcal{F})$ we denote the maximum number $m$ such that each graph of order $n$ and size less than or equal to $m$ has a near-packing admitting $\mathcal{F}$. It is well known that $m\left(n, \mathcal{C}_{0}\right)=m\left(n, \mathcal{D}_{0}\right)=m\left(n, \mathcal{E}_{0}\right)=n-2$ because a near packing admitting $\mathcal{C}_{0}, \mathcal{D}_{0}$ or $\mathcal{E}_{0}$ is just a packing. We prove some generalization of this result, namely we prove that $m\left(n, \mathcal{C}_{k}\right) \approx(k+1) n, m\left(n, \mathcal{D}_{1}\right) \approx \frac{3}{2} n, m\left(n, \mathcal{D}_{2}\right) \approx 2 n$. We also present bounds on $m\left(n, \mathcal{E}_{k}\right)$. Finally, we prove that each graph of girth at least five has a near packing admitting $\mathcal{C}_{1}$ (i.e. a near packing admitting the family of the acyclic graphs).
\end{abstract}

\section{Introduction}

In this paper we use the term graph to refer to simple graphs without loops or multiple edges. The vertex and edge set of a graph $G$ is denoted by $V(G)$ and $E(G)$, respectively. The maximum degree of $G$ is denoted by $\Delta(G)$. A graph is called $k$-connected if any two of its vertices can be joined by $k$ internally vertex disjoint paths. A complete graph $K_{1}$ is

\footnotetext{
*The author was partially supported by the Polish Ministry of Science and Higher Education.
} 
0-connected. By $N_{G}(x)$ we denote the set of vertices adjacent with $x$ in $G$. For a vertex set $X$, the set $N_{G}(X)$ denotes the external neighbourhood of $X$ in $G$, i.e.

$$
N_{G}(X)=\{y \in V(G) \backslash X: y \text { is adjacent with some } x \in X\} .
$$

The degree of a vertex $x$ is the number of vertices adjacent to $x$ and is denoted by $d_{G}(x)$.

Definition 1. Let $G_{1}$ and $G_{2}$ be two graphs such that $\left|V\left(G_{1}\right)\right|=\left|V\left(G_{2}\right)\right|=n$. A packing of $G_{1}$ and $G_{2}$ is a pair of edge-disjoint subgraphs $\left\{H_{1}, H_{2}\right\}$ of $K_{n}$ such that $H_{1} \cong G_{1}$ and $\mathrm{H}_{2} \cong G_{2}$.

Definition 2. Let $\mathcal{F}$ be any family of graphs and let $G_{1}, G_{2}$ be two graphs such that $\left|V\left(G_{1}\right)\right|=\left|V\left(G_{2}\right)\right|=n$. A near packing admitting $\mathcal{F}$ of $G_{1}$ and $G_{2}$ is a pair of subgraphs $\left\{H_{1}, H_{2}\right\}$ of $K_{n}$ such that $H_{1} \cong G_{1}$ and $H_{2} \cong G_{2}$, and the subgraph having edges $E\left(H_{1}\right) \cap$ $E\left(H_{2}\right)$ is a member of $\mathcal{F}$.

Given a graph $G$ and a permutation $\sigma$ of $V(G)$, by $\sigma(G)$ we denote the graph with $V(\sigma(G))=V(G)$ and such that $\sigma(u) \sigma(v) \in E(\sigma(G))$ if and only if $u v \in E(G)$ for any $u, v \in V(G)$. The spanning subgraph of $G$ having edges $E(G) \cap E(\sigma(G))$ is denoted by $G_{\sigma}^{*}$ (abbreviated to $G^{*}$ if no confusion arises). Thus, in case when $G_{1} \cong G_{2} \cong G$ the problem of finding a near packing admitting $\mathcal{F}$ of $G_{1}$ and $G_{2}$ is equivalent to the problem of finding a permutation $\sigma$ of $V(G)$ such that $G_{\sigma}^{*} \in \mathcal{F}$. Such a permutation $\sigma$ of $V(G)$ is called a near packing of $G$ admitting $\mathcal{F}$.

We consider three families of graphs : (1) $\mathcal{E}_{k}$ being the family of all graphs with with at most $k$ edges, (2) $\mathcal{D}_{k}$ being the family of all graphs with maximum degree at most $k$, and (3) $\mathcal{C}_{k}$ being the family of all graphs that do not contain a subgraph of connectivity greater than or equal to $k+1$. Notice that $\mathcal{D}_{0}=\mathcal{C}_{0}=\mathcal{E}_{0}$ is a family of edgeless graphs. Furthermore $\mathcal{C}_{1}$ is a family of acyclic graphs and $\mathcal{C}_{1} \cap \mathcal{D}_{2}$ is a family of linear forests (i.e. disjoint unions of paths).

Let $\mathcal{F}$ be any family of graphs. By $m(n, \mathcal{F})$ we denote the maximum number $m$ such that each graph of order $n$ and size less than or equal to $m$ has a near-packing admitting $\mathcal{F}$. A classic result in this area, obtained independently in $[1,2,7]$, states that

Theorem $3([1,2,7]) \cdot m\left(n, \mathcal{C}_{0}\right)=m\left(n, \mathcal{D}_{0}\right)=m\left(n, \mathcal{E}_{0}\right)=n-2$,

because a near packing admitting $\mathcal{C}_{0}, \mathcal{D}_{0}$ or $\mathcal{E}_{0}$ is just a packing. Our aim is to prove some generalizations of Theorem 3. For every $k \geqslant 1$, we determine $m\left(n, \mathcal{C}_{k}\right)$ up to a constant depending only on $k$. We find the problem concerning near packings admitting $\mathcal{D}_{k}$ considerably harder. We determine only $m\left(n, \mathcal{D}_{1}\right)$ up to a constant, while $m\left(n, \mathcal{D}_{2}\right)$ is determined assymptotically. We also give bounds on $m\left(n, \mathcal{E}_{k}\right)$.

The notion of a near packing was introduced by Eaton [3] in order to obtain some investigations concerning the following conjecture of Bollobás and Eldridge:

Conjecture $4([1])$. If $\left|V\left(G_{1}\right)\right|=\left|V\left(G_{2}\right)\right|=n$ and $\left(\Delta\left(G_{1}\right)+1\right) \cdot\left(\Delta\left(G_{2}\right)+1\right) \leqslant n+1$, then there is a packing of $G_{1}$ and $G_{2}$.

The following theorem is a special case of a more general result proved by Eaton. 
Theorem 5 ([3]). If $\left|V\left(G_{1}\right)\right|=\left|V\left(G_{2}\right)\right|=n$ and $\left(\Delta\left(G_{1}\right)+1\right) \cdot\left(\Delta\left(G_{2}\right)+1\right) \leqslant n+1$, then there is a near packing admitting $\mathcal{D}_{1}$ of $G_{1}$ and $G_{2}$.

We also investigate another conjecture of graph packing by Faudree, Rousseau, Schelp and Schuster [4]:

Conjecture 6. For every non-star graph $G$ of girth at least 5, there is a packing of two copies of $G$.

In particular, Conjecture 6 is true for sufficiently large planar graphs [6]. On the other hand, the statement from the above conjecture is true if $G$ is a non-star graph of girth at least six [5]. In this paper we prove that the statement is true if the term 'packing' is replaced by the term 'near packing admitting $\mathcal{C}_{1}$ '. This result is in some sense best possible, since for every permutation $\sigma$ of $V\left(K_{n, n}\right)$ with $n \geqslant 3, K_{n, n}^{*}$ contains a cycle $C_{4}$.

\section{Preliminaries}

Lemma 7. Let $G$ be a graph and $k, l, q \geqslant 0$ integers. Suppose that $G$ contains an independent set $U \subset V(G)$ that satisfies the following conditions:

1. $d_{G}(u) \leqslant k$ for each $u \in U$,

2. $\left|N_{G}(u) \cap N_{G}(v)\right| \leqslant q$ for every $u, v \in U$.

If $|U| \geqslant \frac{2(k-q)}{l-q+1}$, then for every permutation $\sigma^{\prime}$ of $V(G) \backslash U$ there exists a permutation $\sigma$ of $V(G)$ such that $\left.\sigma\right|_{G-U}=\sigma^{\prime}$ and $d_{G_{\sigma}^{*}}(u) \leqslant l$ for each $u \in U$.

Proof. Let $G^{\prime}:=G-U$ and $\sigma^{\prime}$ be any permutation of $V\left(G^{\prime}\right)$. Below we show that we can extend $\sigma^{\prime}$ to a permutation $\sigma$ as required of $G$.

For any $v \in V\left(G^{\prime}\right)$ let us define $\sigma(v):=\sigma^{\prime}(v)$. Then let us consider a bipartite graph $B$ with partition sets $X:=U \times\{0\}$ and $Y:=U \times\{1\}$. For $u, v \in U$ the vertices $(u, 0)$, $(v, 1)$ are joined by an edge in $B$ if and only if $\left|\sigma^{\prime}\left(N_{G}(u)\right) \cap N_{G}(v)\right| \leqslant l$. So, if $(u, 0),(v, 1)$ are joined by an edge in $B$ we can put $\sigma(u)=v$. In other words, if $(u, 0),(v, 1)$ are not neighbors in $B$, then $\left|\sigma^{\prime}(N(u)) \cap N(v)\right| \geqslant l+1$. Therefore, since $\left|N_{G}(u) \cap N_{G}(v)\right| \leqslant q$ and $d_{G}(u) \leqslant k$ for $u \in U$, we have $d_{B}((u, 0)) \geqslant|U|-\frac{k-q}{l-q+1} \geqslant \frac{k-q}{l-q+1}$, by the assumption on $|U|$. Similarly, $d_{B}((v, 1)) \geqslant \frac{k-q}{l-q+1}$.

Let $S \subset X$. If $|S| \leqslant|U|-\frac{k-q}{l-q+1}$ then obviously $\left|N_{B}(S)\right| \geqslant|S|$. Notice that if $|S|>|U|-\frac{k-q}{l-q+1}$ then $N_{B}(S)=Y$. Indeed, othervise let $(v, 1) \in Y$ be a vertex which has no neighbour in $S$. Thus,

$$
d_{B}((v, 1)) \leqslant|A|-|S|=|U|-|S|<|U|-\left(|U|-\frac{k-q}{l-q+1}\right)=\frac{k-q}{l-q+1},
$$

a contradiction. Hence, in any case $|S| \leqslant|N(S)|$. Thus, by the Hall's theorem there is a matching $M$ in $G$. Therefore we can define $\sigma(u)=v$ for $u, v \in U$ such that $(u, 0),(v, 1)$ are incident with the same edge in $M$. 


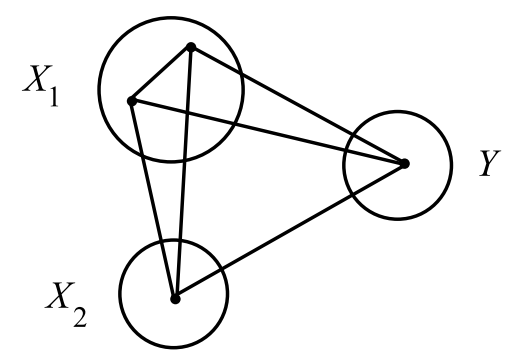

Figure 1: $K_{2,1,1}^{+}$

Proposition 8. Let $G$ be a graph of order $n$ and size $m$ with $m \leqslant$ an $-f(n)$, where $a$ is a real number and $f(n)$ is a non-decreasing function. If $U \subset V(G)$ and vertices from $U$ cover at least $a|U|$ edges, then

$$
m^{\prime} \leqslant a n^{\prime}-f\left(n^{\prime}\right)
$$

where $n^{\prime}$ and $m^{\prime}$ are respectively the order and the size of $G-U$.

Proof.

$$
\begin{aligned}
m^{\prime} & \leqslant a n-f(n)-a|U|=a(n-|U|)-f(n) \\
& \leqslant a(n-|U|)-f(n-|U|)=a n^{\prime}-f\left(n^{\prime}\right),
\end{aligned}
$$

because $f(n) \geqslant f(n-|U|)$.

\section{$3 \quad$ Near packings admitting $\mathcal{C}_{k}$}

Recall that $m\left(n, \mathcal{C}_{0}\right)=n-2$. We start with the following construction. Let $K_{s, k-s, k-s}^{+}$ denote a graph with vertex set $V\left(K_{s, k-s, k-s}^{+}\right)=X_{1} \cup X_{2} \cup Y$ such that $X_{1}, X_{2}, Y$ are pairwise disjoint and $\left|X_{1}\right|=s,\left|X_{2}\right|=|Y|=k-s$. Furthermore, $E\left(K_{s, k-s, k-s}^{+}\right)=E_{1} \cup E_{2}$, where $E_{1}=\left\{x y: x \in X_{1} \cup X_{2}, y \in Y\right\}$ and $E_{2}=\left\{x z: x \in X_{1}, z \in X_{1} \cup X_{2}\right\}$. In other words, $K_{s, k-s, k-s}^{+}$arises from a tripartite graph (with partition sets $X_{1}, X_{2}$ and $Y$ ) by adding all possible edges having two endpoints in $X_{1}$, see Figure 1 . It is easily seen that any two vertices of $K_{s, k-s, k-s}^{+}$are joined by at least $k$ internally vertex disjoint paths, so $K_{s, k-s, k-s}^{+}$is $k$ connected. In what follows $\bar{G}$ denotes the complement of a graph $G$, i.e. a graph on the same vertex set as $G$ and with the property that $e \in E(\bar{G})$ if and only if $e \notin E(G)$.

Lemma 9. $m\left(n, \mathcal{C}_{k}\right) \leqslant(k+1) n-(k+1) \frac{k+2}{2}-1$.

Proof. Let $G=\overline{K_{k+1}}+K_{n-k-1}$. Clearly, $|E(G)|=(k+1) n-(k+1) \frac{k+2}{2}$. We will show that $G$ does not have a near packing admitting $\mathcal{C}_{k}$. Consider an arbitrary permutation $\sigma$ of $V(G)$. Let $S \subset V\left(K_{k+1}\right)$ be a maximal set of vertices with the property that $\sigma(S) \subset V\left(K_{k+1}\right)$. Let $|S|=s$. Then, $G_{\sigma}^{*}$ contains a $K_{s, k+1-s, k+1-s}^{+}$with $X_{1}=S$, $Y=V\left(K_{k+1}\right) \backslash S$ and $X_{2} \subset V\left(K_{n-k-1}\right)$. 
Theorem 10. $m\left(n, \mathcal{C}_{k}\right) \geqslant(k+1) n-4 k(k+1)^{2}-2$.

Proof. For $k=0$ the result follows from Theorem 3. Fix $k \geqslant 1$ and let $c_{k}=4 k(k+1)^{2}+2$. We will prove that each graph of order $n$ and size at most $(k+1) n-c_{k}$ has a near packing admitting $\mathcal{C}_{k}$.

Suppose that $G$ is a counterexample with minimum order $n$. Let $m$ denote the size of $G$, so $m \leqslant(k+1) n-c_{k}$. Note that if $n \leqslant 4(k+1)^{2}$, then

$$
\begin{aligned}
m & \leqslant(k+1) n-c_{k}=k n-c_{k}+n \\
& \leqslant k\left(4(k+1)^{2}\right)-\left(4 k(k+1)^{2}+2\right)+n=n-2 .
\end{aligned}
$$

Hence $G$ has a near packing admitting $\mathcal{C}_{k}$, by Theorem 3, which contradicts our assumption on $G$. Thus, we may assume that $n \geqslant 4(k+1)^{2}+1$. Furthermore, if $\Delta(G) \leqslant 2(k+1)-1$ then $(\Delta(G)+1)^{2} \leqslant 4(k+1)^{2}<n+1$. Hence, $G$ has a near packing admitting $\mathcal{C}_{k}$ by Theorem 5 (because $\mathcal{D}_{1} \subset \mathcal{C}_{k}$ ), a contradiction again. Therefore, we may assume that $\Delta(G) \geqslant 2(k+1)$. Let $w \in V(G)$ with $d_{G}(w) \geqslant 2(k+1)$.

Suppose first that $G$ contains a vertex $u$ with $d_{G}(u) \leqslant k$. By Proposition 8 and by the minimality assumption, $G^{\prime}:=G-\{u, w\}$ has a near packing $\sigma^{\prime}$ admitting $\mathcal{C}_{k}$. We claim that $\sigma:=(u, w) \sigma^{\prime}$ is a near packing of $G$ admitting $\mathcal{C}_{k}$. Indeed, since $d_{G}(u) \leqslant k$ then $d_{G^{*}}(u) \leqslant k$ as well as $d_{G^{*}}(w) \leqslant k$. Hence, neither $u$ nor $w$ can be in a subgraph of $G^{*}$ of connectivity $k+1$ or more. Moreover, since $\left.\sigma\right|_{G^{\prime}}$ is a near packing of $G^{\prime}$ admitting $\mathcal{C}_{k}$, then $G^{*}-\{u, w\}$ does not contain a subgraph of connectivity $k+1$ or more, neither. Therefore, $\sigma$ is a near packing of $G$ admitting $\mathcal{C}_{k}$.

Thus, we may assume that $d_{G}(u) \geqslant k+1$ for every $u \in V(G)$. Let $S$ be a maximum set of vertices of $G$ such that $S$ is independent, $k+1 \leqslant d_{G}(u) \leqslant 2 k+1$ for each $u \in S$, and $\left|N_{G}(u) \cap N_{G}(w)\right| \leqslant k$ for every $u, w \in S$. Since $S$ is independent, by Proposition 8 and by the minimality assumption, $G-S$ has a near packing $\sigma^{\prime \prime}$ admitting $\mathcal{C}_{k}$. By Lemma 7 (with $k, l, q$ replaced by $2 k+1, k, k$, respectively), if $|S| \geqslant 2 k+2$ then there is a permutation $\sigma$ of $G$, such that $\left.\sigma\right|_{G-S}=\sigma^{\prime \prime}$ and $d_{G^{*}}(u) \leqslant k$ for every $u \in S$. Simirarly as before, we can see that $\sigma$ is a near packing of $G$ admitting $\mathcal{C}_{k}$, a contradiction.

Therefore $|S| \leqslant 2 k+1$ and so $\left|N_{G}(S)\right| \leqslant(2 k+1)^{2}$. Let $V_{j}=\left\{v \in V(G) \backslash N_{G}(S)\right.$ : $\left.d_{G}(v)=j\right\}$. Note that by the definition of $S$, we have $\left|N_{G}(S) \cap N_{G}(u)\right| \geqslant k+1$ for every $u \in V_{k+1} \cup \cdots \cup V_{2 k+1}$. Hence, vertices from $N_{G}(S)$ are incident (in common) to at least $(k+1)\left(\left|V_{k+1}\right|+\cdots+\left|V_{2 k+1}\right|\right)$ edges. Thus,

$$
\begin{aligned}
(2 k+2) n- & 8 k(k+1)^{2}-4 \geqslant 2 m \\
= & \sum_{u \in N_{G}(S)} d_{G}(u)+\sum_{v \in V(G) \backslash N_{G}(S)} d_{G}(v) \\
\geqslant & (k+1)\left(\left|V_{k+1}\right|+\cdots\left|V_{2 k+1}\right|\right)+(k+1)\left|V_{k+1}\right|+\cdots+(2 k+1)\left|V_{2 k+1}\right| \\
& +(2 k+2)\left(n-\left|V_{k+1}\right|+\cdots\left|V_{2 k+1}\right|-\left|N_{G}(S)\right|\right) \\
\geqslant & (2 k+2) n-(2 k+2)(2 k+1)^{2},
\end{aligned}
$$

a contradiction. Hence, we deduce no counterexample to Theorem 10 exists. 
Theorem 11. Every graph of girth at least 5 has a near packing admitting $\mathcal{C}_{1}$.

Proof. Let $G$ be a minimum counterexample to Theorem 11. Let $u \in V(G)$ with $d_{G}(u)=$ $\Delta(G)$. Let $G^{\prime}=G-u$ and $U=N_{G}(u)$. By the girth assumption, $U$ is an independent set in $G^{\prime}$ (as well as in $G$ ), and $N_{G^{\prime}}(x) \cap N_{G^{\prime}}(y)=\emptyset$ for every $x, y \in U$. By the minimality assumption $G^{\prime \prime}:=G^{\prime}-U$ has a near packing $\sigma^{\prime \prime}$ admitting $\mathcal{C}_{1}$. Moreover, $|U|=\Delta(G)$ and $d_{G^{\prime}}(u) \leqslant \Delta(G)-1$. Hence, by Lemma 7 (with $k=\Delta(G)-1, l=1, q=0$ ), $G^{\prime}$ has a near packing $\sigma^{\prime}$ such that $\left.\sigma^{\prime}\right|_{G^{\prime \prime}}=\sigma^{\prime \prime}$ and $d_{G^{\prime *}}(u) \leqslant 1$ for each $u \in U$. Thus, since $G^{\prime \prime *}$ is acyclic, $G^{\prime *}$ is also acyclic. Let $u$ be any vertex from $U$. It is easy to see that the permutation $\sigma$ such that $\sigma(u)=x, \sigma(x)=u$ and $\sigma(y)=\sigma^{\prime}(y)$ for every $y \in V(G) \backslash\{u, x\}$ is a near packing of $G$ admitting $\mathcal{C}_{1}$, a contradiction.

\section{Near packings admitting $\mathcal{D}_{k}$}

Recall that $m\left(n, \mathcal{D}_{0}\right)=n-2$.

Lemma 12. $m\left(n, \mathcal{D}_{k}\right) \leqslant\left\lceil\frac{(k+2)(n-1)}{2}\right\rceil-1$.

Proof. Let $H$ be a $k$-regular graph of order $n-1$ provided that $k$ is even or $n-1$ is even. Otherwise, let $H$ be a graph with all but one vertices having degree $k$ and one vertex having degree $k+1$. Let $G=K_{1}+H$ and $V\left(K_{1}\right)=\{u\}$. It is easily seen that for any permutation $\sigma$ of $V(G)$, the vertex $u$ (as well as its image) has degree at least $k+1$ in $G_{\sigma}^{*}$. Thus, $G$ does not have a near packing admitting $\mathcal{D}_{k}$. Furthermore, $E(G)=\frac{(k+1)(n-1)+n-1}{2}=\frac{(k+2)(n-1)}{2}$ if $k$ is even or $n-1$ is even, or $E(G)=\frac{(k+1)(n-2)+(k+2)+(n-1)}{2}=\frac{(k+2)(n-1)+1}{2}$ otherwise.

We are tempted to propose the following conjecture

\section{Conjecture 13.}

$$
\frac{k+2}{2} n-c_{1}(k) \leqslant m\left(n, \mathcal{D}_{k}\right) \leqslant \frac{k+2}{2} n-c_{2}(k),
$$

where $c_{i}(k)$ are constants depending only on $k$.

The next theorem confirms Conjecture 13 for $k=1$.

Theorem 14. $m\left(n, \mathcal{D}_{1}\right) \geqslant \frac{3}{2} n-10$.

Proof. Let $G$ be a counterexample of minimum order $n$. Without loss of generality we assume that $m:=|E(G)|=\frac{3}{2} n-10$. Note that if $n \leqslant 16$ then $\frac{3}{2} n-10 \leqslant n-2$. Thus, by Theorem 3, $G$ has a packing which contradicts our assumption on $G$. Hence, we may assume that $n \geqslant 17$. Furthermore, if $\Delta(G) \leqslant 3$, then $(\Delta(G)+1)^{2} \leqslant 16<n+1$, so $G$ has a near packing admitting $\mathcal{D}_{1}$ by Theorem 5 . Thus, we may assume that $\Delta(G) \geqslant 4$. Let $w \in V(G)$ with $d_{G}(w) \geqslant 4$.

Suppose first that $G$ has a vertex $u$ with $d_{G}(u)=0$. Then, by Proposition 8 and by the minimality assumption, $G_{1}:=G-\{u, w\}$ has a a near packing $\sigma_{1}$ admitting $\mathcal{D}_{1}$. Clearly, $(u, w) \sigma_{1}$ is a near packing of $G$ admitting $\mathcal{D}_{1}$. 
So we may assume that $G$ has no isolated vertex. Suppose now that $G$ has a vertex $u$ with $d_{G}(u)=1$ and let $v$ be the neighbor of $u$. If $d_{G}(v) \geqslant 3$ then, by Proposition 8 and by the minimality assumption, $G_{2}:=G-\{u, v\}$ has a near packing $\sigma_{2}$ admitting $\mathcal{D}_{1}$. Clearly, $(u, v) \sigma_{2}$ is a near packing admitting $\mathcal{D}_{1}$ of $G$. Similarly, if $d_{G}(v)=1$ then $(u)(w, v) \sigma_{3}$ is a near packing admitting $\mathcal{D}_{1}$ of $G$ where $\sigma_{3}$ is a near packing admitting $\mathcal{D}_{1}$ of $G-\{u, v, w\}$ ( $\sigma_{3}$ exists by the minimality assumption). Thus we may assume that $d_{G}(v)=2$. Let $x$ be the neighbor of $v$ different from $u$. If $x \neq w$ then $(u)(v, w, x) \sigma_{4}$ is a near packing admitting $\mathcal{D}_{1}$ of $G$ where $\sigma_{4}$ is a near packing admitting $\mathcal{D}_{1}$ of $G-\{u, v, w, x\}$ ( $\sigma_{4}$ exists by the minimality assumption). Finally, if $x=w$ then $(u)(v, w) \sigma_{5}$ is a near packing admitting $\mathcal{D}_{1}$ of $G$ where $\sigma_{5}$ is a near packing admitting $\mathcal{D}_{1}$ of $G-\{u, v, w\}\left(\sigma_{5}\right.$ exists by the minimality assumption).

Therefore, we may assume that $d_{G}(u) \geqslant 2$ for each $u \in V(G)$. Let $S \subset V(G)$ be a maximal set such that $S$ is independent in $G, d_{G}(v)=2$ for every $v \in S$, and $N_{G}(u) \cap N_{G}(v)=\emptyset$ for every $u, v \in S$. Note that $S \neq \emptyset$. By Proposition 8 and by the minimality assumption, $G-S$ has a near packing $\sigma^{\prime}$ admitting $\mathcal{D}_{1}$. Note that if $|S| \geqslant 4$, then by Lemma 7 (with $k=2, q=0$ and $l=0$ ), there exists a near packing of $G$ admitting $\mathcal{D}_{1}$, a contradiction with the assumption on $G$. Thus, $|S| \leqslant 3$ and so $\left|N_{G}(S)\right| \leqslant 6$. Let $V_{j}=\left\{v \in V(G) \backslash N_{G}(S): d_{G}(v)=j\right\}$. Note that by the definition of $S$, we have $\left|N_{G}(S) \cap N_{G}(u)\right| \geqslant 1$ for every $u \in V_{2}$. Therefore,

$$
\begin{aligned}
3 n-20 & =2 m=\sum_{u \in N_{G}(S)} d_{G}(u)+\sum_{v \in V(G) \backslash N_{G}(S)} d_{G}(v) \\
& \geqslant\left|V_{2}\right|+2\left|V_{2}\right|+3\left(n-\left|V_{2}\right|-\left|N_{G}(S)\right|\right) \geqslant 3 n-18,
\end{aligned}
$$

a contradiction. Hence, we deduce no counterexample to Theorem 14 exists.

The following result provides some evidence for Conjecture 13 in case when $k=2$.

Theorem $15([8]) \cdot m\left(n, \mathcal{D}_{2}\right) \geqslant 2 n-10 n^{2 / 3}-7$.

\section{$5 \quad$ Near packings admitting $\mathcal{E}_{k}$}

The join $G=G_{1}+G_{2}$ of graphs $G_{1}$ and $G_{2}$ with disjoint vertex sets $V_{1}$ and $V_{2}$ and edge sets $E_{1}$ and $E_{2}$ is the graph union $G_{1} \cup G_{2}=\left(V_{1} \cup V_{2}, E_{1} \cup E_{2}\right)$ together with all the edges joining $V_{1}$ and $V_{2}$.

Lemma 16. If $n \geqslant 2 k+2$ then $m\left(n, \mathcal{E}_{2 k}\right) \leqslant\left\lceil\frac{(k+2)(n-1)}{2}\right\rceil-1$.

Proof. Let $H$ be a $k$-regular graph of order $n-1$ provided that $k$ is even or $n-1$ is even. Otherwise, let $H$ be a graph with all but one vertices having degree $k$ and one vertex having degree $k+1$. Let $G=K_{1}+H$ and $V\left(K_{1}\right)=\{u\}$. It is easily seen that for any permutation $\sigma$ of $V(G)$, the vertex $u$ as well as $\sigma(u)$ has degree at least $k+1$ in $G_{\sigma}^{*}$. Thus, if $u \neq \sigma(u)$ then $G_{\sigma}^{*}$ has at least $2 k+1$ edges. If $u=\sigma(u)$ then $u$ has degree $n-1$ in $G_{\sigma}^{*}$. Since $n \geqslant 2 k+2, G_{\sigma}^{*}$ has at least $2 k+1$ edges. Therefore, $G$ does not have a near 
packing admitting $\mathcal{E}_{2 k}$. Furthermore, $E(G)=\frac{(k+1)(n-1)+n-1}{2}=\frac{(k+2)(n-1)}{2}$ if $k$ is even or $n-1$ is even, or $E(G)=\frac{(k+1)(n-2)+(k+2)+(n-1)}{2}=\frac{(k+2)(n-1)+1}{2}$ otherwise.

Theorem 17. $m\left(n, \mathcal{E}_{k}\right) \geqslant \sqrt{\frac{k}{2} n(n-1)}$.

Proof. Let $G$ be a graph of order $n$ and size $m$. We will prove that if $m \leqslant \sqrt{\frac{k}{2} n(n-1)}$ then there is a near-packing of $G$ admitting $\mathcal{E}_{k}$. Consider the probability space whose $n$ ! points are the permutations of $V(G)$. For any two edges $e, f \in E(G)$ let $X_{e f}$ denote the indicator random variable with value 1 if $f$ is an image of $e$. Then

$$
E\left(X_{e f}\right)=\operatorname{Prob}\left(X_{e f}=1\right)=\frac{2(n-2) !}{n !}=\left(\begin{array}{l}
n \\
2
\end{array}\right)^{-1}
$$

Let $X=\sum_{e, f \in E(G)} X_{e f}$. Thus, by the linearity of expectation, we have

$$
E(X)=\sum_{e, f \in E(G)} E\left(X_{e f}\right) \leqslant m^{2}\left(\begin{array}{l}
n \\
2
\end{array}\right)^{-1} \leqslant k .
$$

This implies that there exists a permutation $\sigma$ of $V(G)$ such that $G_{\sigma}^{*}$ has at most $k$ edges. Thus, $\sigma$ is a near packing of $G$ admitting $\mathcal{E}_{k}$.

\section{References}

[1] B. Bollobás and S. E. Eldridge, Packing of graphs and applications to computational complexity, J. Combin. Theory Ser. B 25:105-124, 1978.

[2] D. Burns and S. Schuster, Every $(p, p-2)$ graph is contained in its complement, $J$. Graph Theory 1:277-279, 1977.

[3] N. Eaton, A near packing of two graphs, J. Combin. Theory Ser. B, 80:98-103, 2000.

[4] R. J. Faudree, C. C. Rousseau, R. H. Schelp, and S. Schuster, Embedding graphs in their complements, Czechoslovak Math. J., 31(106):53-62, 1981.

[5] A. Görlich and A. Żak, A note on packing graphs without cycles of length up to five, Electronic J. Combin., 16:N30, 2009.

[6] A. Görlich and A. Żak, Sparse graphs of girth at least five are packable, Discrete Math., 312:3606-3613, 2012.

[7] N. Sauer and J. Spencer, Edge disjoint placement of graphs, J. Combin. Theory Ser. B, 25:295-302, 1978.

[8] A. Żak, unpublished, 4 pages. 